\title{
ON THE ORIGIN OF IO'S ULTRAVIOLET AURORA
}

\author{
V. E. Shaposhnikov*, V. V. Zaitsev*, and H. O. Rucker ${ }^{\dagger}$
}

\begin{abstract}
A model involving an additional contribution to Io's ultraviolet (UV) aurora is presented. A mechanism for heating of electrons of Io's ionospheric plasma up to sufficient energies for the excitation of Io's atmospheric oxygen and emitting of observed UV emission is proposed. The mechanism operates by the effect of the different magnetization of the electrons and ions in Io's ionosphere which in the course of Io's motion through the Jovian magnetic field causes the creation of a charge-separation electric field in the upstream part of the ionosphere. This field has a component parallel to the magnetic and shifts the electron distribution function relative to the ion distribution function by a value exceeding the thermal velocity of electrons. In this case, a Bunemann instability with a very large growth rate develops. This results in the excitation of turbulent pulsations at frequency close to the ion-sound frequency and the occurrence of anomalous resistance to the electric current. The latter causes heating of Io's ionospheric electrons up to a temperature of about $25 \mathrm{eV}$. Atmospheric oxygen molecules excited by collisions with the heated electrons of Io's ionosphere, whose density is about $6 \times 10^{4} \mathrm{~cm}^{-3}$, can contribute to the observed UV brightness. The proposed model permits one to explain the correlation of UV brightness with Io's magnetic longitude and the discrepancy between the anti-Jovian equatorial UV spots and sub-Jovian spots as well.
\end{abstract}

\section{Introduction}

The Jovian satellite Io plays an important role in many processes occurring in the Jovian magnetosphere and ionosphere. Io is surrounded by an atmosphere, and knowledge of its composition and properties is necessary for the understanding of interaction between the satellite and the Jovian magnetosphere. Important information on the atmosphere comes from observations in the ultraviolet (UV) wavelength range. Papers [Ballester et

\footnotetext{
*Institute of Applied Physics, Russian Academy of Science, 46 Ul'yanov Street, 603950, Nizhni Novgorod, Russia

† Space Research Institute, Austrian Academy of Sciences, Schmiedlstrasse 6, A-8042 Graz, Austria
} 
al., 1987; Clarke et al., 1994; Durrance et al., 1995; Fox et al., 1997] report on observations of Io and its vicinity in the UV lines of oxygen OI and sulfur SI atoms. However, small spatial resolution precluded the study of the spatial structure of this radiation in these experiments. The situation changed after the Space Telescope Imaging Spectrograph (STIS) was mounted on the Hubble space telescope. Even the first observations of UV emission from Io's atmosphere performed with the use of STIS displayed very interesting results [Roesler et al., 1999; Saur et al., 2000; Retherford et al., 2000]. Two brightest sources of UV emission above Io's limb near its equator (which they called "equatorial spots", Fig. 1) were observed. The sources are about $200 \mathrm{~km}$ above Io's surface and extend several hundred kilometers above this altitude. Their position is shifted with the Jovian magnetic field orientation at Io. The brightness of the equatorial spots can reach 2500 Rayleigh in OI $1356 \mathrm{~A}$ line and the anti-Jovian equatorial spots are $20 \%$ brighter than the sub-Jovian equatorial spots. It is also found that the brightness of the emissions is correlated with Io's magnetic longitude and, therefore, also with Io's distance from the plasma torus centrifugal equator.

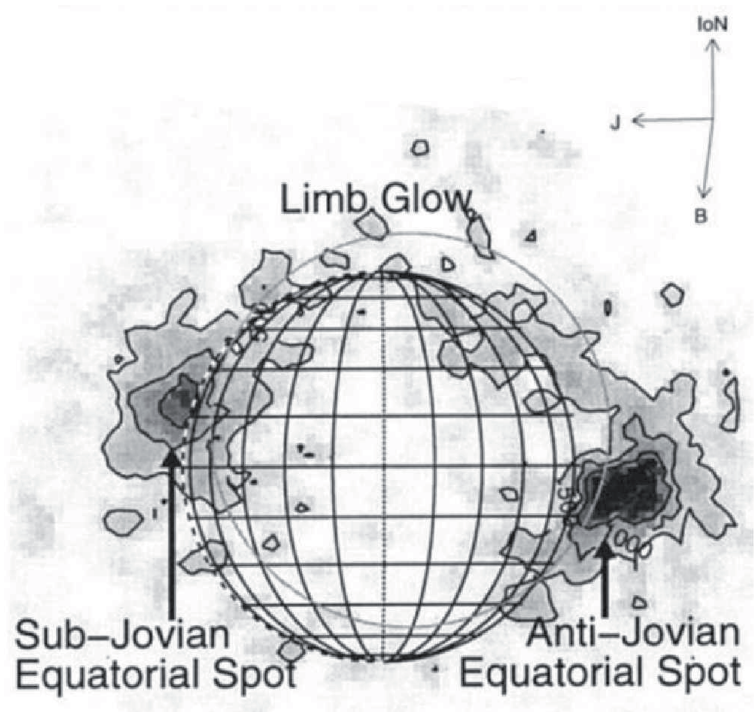

Figure 1: STIS image of OI 1356 Aemission October 14, 1997. The picture is adapted from Retherford et al. [2000].

Saur et al. [2000] proposed a model for explaining the features of the equatorial spots. For their analysis they used oxygen OI $1356 \AA$ because it is representative of the radiation patterns of other observed oxygen and sulfur multiplets. According to their model, the electrons from Io's plasma torus are convected into Io's atmosphere and excite neutral oxygen and sulfur due to inelastic collisions. This mainly takes place on the flanks and the upstream side. The maximum number of collisions and maximum UV emission occur on Io's flanks where the atmospheric density is maximum. Their model also gives an 
acceptable explanation for why the anti-Jovian equatorial spots are brighter than the sub-Jovian equatorial spots and why the equatorial spot location are correlated with the Jovian magnetic fields orientation. Owning to the anisotropic conductivity of ionosphere and to the induced Hall electric field, the electrons on the sub-Jovian side before reaching the sub-Jovian flanks, traverse the upstream atmosphere where they lost part of their energy, while the electrons on the anti-Jovian side deposit their energy directly on the flank. This produces the observed brightness asymmetry. However, they could not explain the observed brightness of the equatorial spots. Their model yields a peak intensity of $\sim$ 130 Rayleigh only in comparison to the observed maximum intensity of $\sim 2500$ Rayleigh. To match the observed brightness with the model one, Saur et al. [2000] conclude that the mixing ratio $[\mathrm{O}] /\left[\mathrm{SO}_{2}\right]$ must be $\sim 20 \%$ in the upper atmosphere, which is much higher than the ratio predicted $(\sim 1 \%)$ with the diffusive photochemical model by Summers and Strobel [1996].

We believe that the problem of the model of Saur et al. [2000] is that the temperature of electrons which penetrate on the flanks of Io's atmosphere and collide with neutral oxygen and sulfur is too low. It is no more than $5 \mathrm{eV}$ [Saur et al., 1999], while the transition energy is about $10 \mathrm{eV}$ in the UV lines. That is why the electrons of a high-energy tail of the electron distribution function contribute into the UV emission only. Note here that the high density of plasma observed in the Io's wake required a supplemental ionization source beyond the thermal electrons of the Io torus [Dols et al., 2008].

In the next section, we will show that an effective mechanism of heating of the ionospheric electrons up to sufficient energies for the effective excitation of Io's atmospheric atoms and generation of the observed UV radiation is realized in Io's ionosphere.

\section{Heating Mechanism of Ionospheric Electrons and Generation of UV Emission}

The electrodynamic interaction between Jupiter and its satellite Io occurs mainly in a rather dense Io ionosphere, which is separated from the surface by a layer of neutral gas (regions 2 and 1 in Fig. 2). The motion of Io induces an electric field in the Io ionosphere $\vec{E}_{\mathrm{i}}=(1 / c)\left[\vec{V} \vec{B}_{0}\right]$ where $\vec{B}_{0}$ is the Jovian magnetic field at Io's orbit, $V$ is Io's velocity with respect to the co-rotating Jovian magnetospheric plasma, and $\mathrm{c}$ is the speed of light. This electric field cannot directly accelerate the particles since it is perpendicular to the magnetic field. However, due to the anisotropic conductivity of the satellite ionosphere, the electric field $\vec{E}_{\mathrm{i}}$ induces Pedersen electric currents along $\vec{E}_{\mathrm{i}}$ and tends to generate also Hall currents whose direction in the ionosphere of Io's upstream side is approximately orthogonal to the moon surface. Hall currents cannot be closed through the surface due to the neutral atmosphere near the surface. Therefore, a considerable separation of charges occurs in Io's ionosphere. In Io's ionosphere, the electrons are magnetized more stronger than the ions, namely, $\omega_{\mathrm{e}}^{2} \tau_{\mathrm{e}}^{2} \gg 1$ and $\omega_{\mathrm{e}} \tau_{\mathrm{e}} \gg \omega_{\mathrm{i}} \tau_{\mathrm{i}}\left(\omega_{\mathrm{e}}\right.$ and $\omega_{\mathrm{i}}$ are the electron and ion gyrofrequencies, respectively, $\tau_{\mathrm{e}}=\left(\nu_{\mathrm{ei}}+\nu_{\mathrm{en}}\right)^{-1}$ and $\tau_{\mathrm{i}}=\nu_{\mathrm{in}}^{-1}, \nu_{\mathrm{ei}}$, and $\nu_{\mathrm{en}}$ are the frequencies of collisions of electrons (e) with ions (i) and neutrals (n), $\nu_{\text {in }}$ is the frequency collisions of ions (i) with neutrals (n)). The longitudinal component of the electric field is 


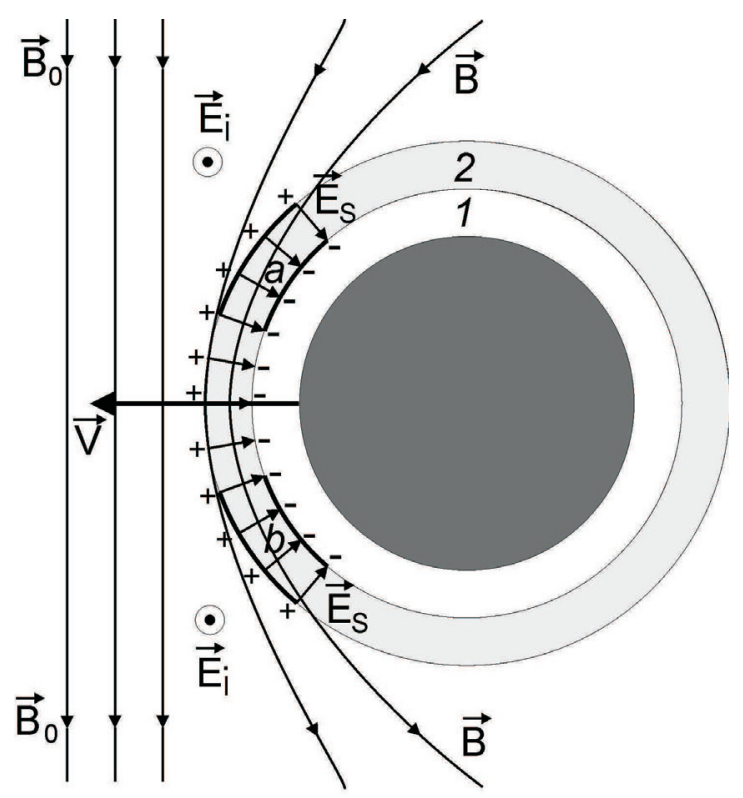

\section{1 - neutral atmosphere}

\section{2 - ionosphere}

Figure 2: Field system generated by the motion of Io through the planetary magnetic field $\vec{B}_{0}$.

maximum when the incoming planetary magnetic field lines are almost tangential to Io's surface [Zaitsev et al., 2003]. In this case,

$$
E_{\|}=0.5 \frac{V B}{c}
$$

where $B$ is the planetary magnetic field near Io.

The electric field $E_{\|}$accelerates all ionospheric electrons till the moment when the force $e E_{\|}$is placed in equilibrium with the fraction force caused by collisions between electrons and neutral particles. According to the estimates made by Zaitsev et al. [2003], the arising mean electron velocity appears to be greater than the thermal electron velocity. This leads to the onset of Bunneman instability and additional heating of electrons. The most efficient channel for electron cooling in Io's ionosphere is the electron thermal conductivity along the magnetic field. The electron temperature is determined from the condition of a balance between the electron heating (due to the Bunneman instability) and the rate of cooling down (due to the electron heat conductivity). For the parameters at the maximum of Io's ionosphere, namely, the electron density $n_{\mathrm{e}} \simeq 4 \times 10^{4} \mathrm{~cm}^{-3}$, the density of neutral particles at the maximum of the ionosphere $n_{\mathrm{m}} \simeq 4 \times 10^{10} \mathrm{~cm}^{-3}$, and the electron temperature $T_{\mathrm{e}}>0.1 \mathrm{eV}$, we find that the temperature of heated electrons can 
reach [Zaitsev et al., 2003, 2006] $T_{\mathrm{h}} \geq 25 \mathrm{eV}$. As an example, Fig. 3 shows the function of oxygen excitation in the UV line OI $1356 \AA$. It is seen in this figure that for $T_{\mathrm{h}} \geq 25 \mathrm{eV}$ the excitation cross section is high enough and the heated ionospheric electrons can effectively excite ultraviolet lines of oxygen.

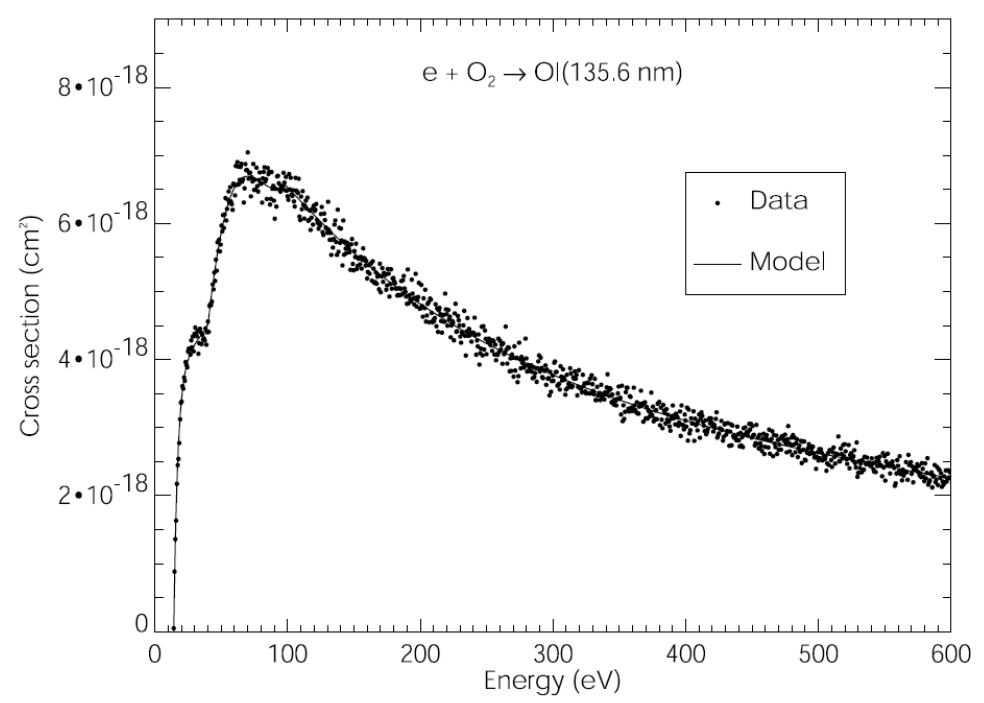

Figure 3: The OI 1356 A excitation function [Kanik et al., 2003].

In crossed electric $E_{\mathrm{i}}$ and magnetic B fields, the heated ionospheric electrons are mixed into Io's flanks. The electrons collide with the atoms and molecules of Io's atmosphere and create UV aurora. Using the excitation cross section presented in Fig. 3, it is easy to estimate the UV photon flux in the OI 1356 Aline. The choice of the line is also convenient because in this case the transitions are semi-forbidden and, therefore, the optical depth of the source is less than unity, i.e., the source is optically thin [Saur et al., 2000]. In this case, the UV photon flux in such collisions can easily be estimated from the formula

$$
F=\sigma(E) n_{\mathrm{h}} n_{\mathrm{m}} \sqrt{\frac{2 E}{m_{\mathrm{e}}}} L \text { photons } / \mathrm{cm}^{2} \mathrm{~s},
$$

where $\sigma(E)$ is the electron impact cross section, $E$ is the energy of electrons, $n_{\mathrm{h}}$ is the density of hot electrons, $n_{\mathrm{a}}$ is the density of oxygen atoms, $m_{\mathrm{e}}$ is the electron mass, and $\mathrm{L}$ is a typical size of the region of collisions along the line of sight. Assuming for estimates that the abundance of oxygen atoms in the Io's ionosphere is $1 \%$, the mean column density of Io's atmosphere is $10^{16} \mathrm{~cm}^{-2}$, and the temperature of hot electrons is $25 \mathrm{eV}$, we find that the the brightness of an ultraviolet source on Io's flanks can reach $F \simeq 2800$ Rayleigh. The obtained value is in good agreement with the observed maximum intensity, which is $\sim 2500$ Rayleigh. 


\section{Discussion}

In the previous section we showed that the heating of electrons in Io's ionosphere due to the electric field of charge separation makes it possible to increase the electron energy up to values sufficient for the effective excitation of neutral atoms of the ionosphere and generation of the observed UV radiation from the sources on Io's flanks.

As is shown in Zaitsev et al. [2003], the electric field of charge separation and the heating of ionospheric electrons occur on the upstream side of Io, where the planetary magnetic field lines are almost tangential to Io's surface. This condition is fulfilled in the near-equator regions of Io's ionosphere. It was mentioned in the previous sections that the electrons heated on the upstream side of Io in crossed electric and magnetic fields shift towards the satellite flanks with velocity approximately equal to the velocity of the incident plasma flux of Io's torus. At the same time, a cloud of heated electrons extends along the magnetic field lines with the ambipolar diffusion. Estimates show that this velocity is much lower than the velocity of plasma shift towards the satellite flanks. Hence, the electrons heated on the flanks and the UV sources stipulated by these electrons turn out to be concentrated near the equator, namely in the region where the magnetic field is almost tangential to the satellite surface and change their position with the variation in the orientation of the local Jovian magnetic field. Moreover, the electric field of charge separation and the heating of electrons are proportional to the local planetary magnetic field on the upstream side of Io's ionosphere. Since the value of the planetary magnetic field along Io's orbit changes with the variation in Io's magnetic longitudes, the heating of ionospheric electrons changes as well. Therefore, the brightness of the equatorial UV sources appears to be correlated with Io's magnetic longitudes and with Io's distance from the centrifugal equator of the plasma torus.

The Hall electric field not only stipulates the occurrence of the electric field of charge separation and the heating of electrons, but also a variation in the direction of motion of heated electrons in crossed electric and magnetic fields towards Jupiter. As a result, the electrons on the anti-Jovian side of Io move on the flanks closer to Io's surface, i.e., in the denser layers of the atmosphere than the electrons on the sub-Jovian side. According to Eq. 2, the brightness of an anti-Jovian UV source turns out to be higher than the brightness of a sub-Jovian source.

The brightness of UV emission is proportional to the length of the region with the maximum number of collisions along the line of sight (see Eq. 2). This length is much greater on Io's flanks than on the upstream side. In the latter case, this length is determined by the scale of the ionosphere height and does not exceed $100 \mathrm{~km}$. Therefore, the brightness of UV emission from the equatorial spots is significantly higher than the UV emission from the upstream side of Io's ionosphere.

According to Zaitsev et al. [2003, 2006], the heating is the maximum in the region of the maximum concentration Io's ionospheric electrons. As the distance from Io's surface increases, the temperature of heated electrons and, therefore, the brightness of the UV emission caused by collisions of these electrons with neutrals decrease. Beginning with a certain altitude, the contribution of heated electrons into the UV emission of equatorial spots becomes smaller than the contribution of the Io plasma torus electrons penetrating 
into the atmosphere (see Saur et al. [2000]). It is exactly the electron fluxes of Io's plasma torus penetrating into Io's atmosphere that determine the large extension of UV spots in altitude.

Acknowledgments. The work was supported by the Russian Foundation for Basic Research (project Nos. 08-02-00119-a, 11-02-00103-a, and 09-02-90406-Ukr-f-a), the General Physics Division of the Russian Academy of Sciences (the Basic Research Program "Plasma Processes in the Solar System"), and the Academic exchange program of the Austrian Academy of Sciences.

\section{References}

Ballester, G. E., H. W. Moos, P. D. Feldman, D. F. Strobel, M. E. Summers, J.-L. Bertaux, T. E. Skinner, M. C. Festou, and J. H. Lieske, Detection of neutral oxygen and sulfur emissions near Io using IUE, Astrophys. J., 319, L33-L38, 1987.

Clarke, J. T., J. Ajello, J. Luhmann, N. Schneider, and I. Kanik, Hubble Space Telescope UV spectral observations of Io passing into eclipse, J. Geophys. Res., 99, 8387-8402, 1994.

Dols, V., P. A. Delamere, and F. Begenal, A multispecies chemestry model of Io's local interaction with the plama torus, J. Geophys. Res., 113, A09208, 2008.

Fox, G. K., A.D. Code, C. M. Anderson, B. L. Babler, K.S. Bjorkman, J. J. Johnson, M. R. Meade, K. H. Nordsieck, W. T. Sanders, A. J. Weitenbeck, N. E. B. Zellner, R. J. Edgar, and O. L. Lupie, Solar System Observations by the Wisconsin Ultraviolet Photopolarimeter Experiment. II. The First Linear Ultraviolet Spectropolarimetry of Io, Astronom. J., 113, 1158, 1997.

Kanik, I., C. Noren, O.P. Makarov, P. Vattipalle, and J. M. Ajello, Electron impact dissociative excitation $\mathrm{O}_{2}$ : 2. Absolute emission cross section of the $\mathrm{OI}(130.4 \mathrm{~nm})$ and OI(135.6 nm) lines, J. Geophys. Res., 108, 5126, 2003.

Retherford, K. D., H. W. Moors, D. F. Strobel, B. C. Wolven, and F. L. Roesler, Io's equatorial spots: Morphology of neutral UV emissions, J. Geophys. Res., 105, 2715727165, 2000.

Roesler, F. L., H.W. Moors, R. J. Oliversen, R. C. Woodward Jr., K. D. Retherford, F. Scherb, M. A. McGrath, W. H. Smyth, P.D. Feldman, and D. F. Strobel, Farultraviolet imaging spectroscopy of Io's atmosphere with HST/STIS, Science, 283, 353-357, 1999.

Summers, M. E. and D. F. Strobel, Photochemestry and vertical transport in Io's atmosphere and ionosphere, Icarus, 120, 290-316, 1996.

Saur, J., F. Neubauer, D.F. Strobel, and M.E. Summers, Three-dimensional plasma simulation of Io's interaction with the Io plasma torus: Asummetric plasma flow, J. Geophys. Res., 104, 25105-25126, 1999. 
Saur, J., F. Neubauer, D. F. Strobel, and M. E. Summers, Io's ultraviolet aurora: Remote sensing of Io's, Geophys. Res. Lett., 27, 2893-2896, 2000.

Zaitsev, V.V., V.E. Shaposhnikov, and H. O. Rucker, Electron acceleration in the ionosphere of Io, Astron. Rep., 80, 761-768, 2003.

Zaitsev, V.V., V.E. Shaposhnikov, and H. O. Rucker, Dependence of the Io-related decametric radio emission of Jupiter on the central maredian longitude and Io's "active" longitudes, Astron. Astrophys., 454, 669-676, 2006. 